\title{
Evaluation of alternative mosquito sampling methods for malaria vectors in Lowland South - East Zambia
}

Chadwick H Sikaala ${ }^{1,2^{*}}$, Gerry F Killeen ${ }^{2,3}$, Javan Chanda ${ }^{1}$, Dingani Chinula ${ }^{1}$, John M Miller ${ }^{4}$, Tanya L Russell ${ }^{2,3,5}$ and Aklilu Seyoum ${ }^{2}$

\begin{abstract}
Background: Sampling malaria vectors and measuring their biting density is of paramount importance for entomological surveys of malaria transmission. Human landing catch (HLC) has been traditionally regarded as a gold standard method for surveying human exposure to mosquito bites. However, due to the risk of human participant exposure to mosquito-borne parasites and viruses, a variety of alternative, exposure-free trapping methods were compared in lowland, south-east Zambia.

Methods: Centres for Disease Control and Prevention miniature light trap (CDC-LT), Ifakara Tent Trap model C (ITT-C), resting boxes (RB) and window exit traps (WET) were all compared with HLC using a $3 \times 3$ Latin Squares design replicated in 4 blocks of 3 houses with long lasting insecticidal nets, half of which were also sprayed with a residual deltamethrin formulation, which was repeated for 10 rounds of 3 nights of rotation each during both the dry and wet seasons.

Results: The mean catches of HLC indoor, HLC outdoor, CDC-LT, ITT-C, WET, RB indoor and RB outdoor, were 1.687, $1.004,3.267,0.088,0.004,0.000$ and 0.008 for Anopheles quadriannulatus Theobald respectively, and 7.287, 6.784, 10.958, 5.875, 0.296, 0.158 and 0.458 , for An. funestus Giles, respectively. Indoor CDC-LT was more efficient in sampling An. quadriannulatus and An. funestus than HLC indoor (Relative rate [95\% Confidence Interval] $=1.873[1.653,2.122]$ and 1.532 [1.441, 1.628], respectively, $P<0.001$ for both). ITT-C was the only other alternative which had comparable sensitivity (RR $=0.821[0.765,0.881], P<0.001)$, relative to HLC indoor other than CDC-LT for sampling An. funestus.

Conclusions: While the two most sensitive exposure-free techniques primarily capture host-seeking mosquitoes, both have substantial disadvantages for routine community-based surveillance applications: the CDC-LT requires regular recharging of batteries while the bulkiness of ITT-C makes it difficult to move between sampling locations. RB placed indoors or outdoors and WET had consistently poor sensitivity so it may be useful to evaluate additional alternative methods, such as pyrethrum spray catches and back packer aspirators, for catching resting mosquitoes.
\end{abstract}

Keywords: Anopheles quadriannulatus, Anopheles funestus, Sampling, Ifakara Tent Trap, Sensitivity

\footnotetext{
* Correspondence: chsikaala@yahoo.co.uk

${ }^{1}$ National Malaria Control Centre, Chainama Hospital College Grounds, Off

Great East road, P.O. Box 32509, Lusaka, Zambia

${ }^{2}$ Vector Biology Department, Liverpool School of Tropical Medicine,

Pembroke Place, Liverpool L3 5QA, UK

Full list of author information is available at the end of the article
} 


\section{Background}

In measuring malaria transmission intensity under varying epidemiological settings, entomological sampling methods that catch mosquitoes with high sensitivity are very useful, particularly as vector densities drop in response to increasingly effective vector control and elimination of transmission is prioritised by an increasing number of countries [1-5]. Generally, these sampling methods involve collection of adult mosquitoes either indoors or outdoors, with the host-seeking females that mediate transmission as the primary target for trapping [5,6]. Human landing catch (HLC) is the gold standard method for collection of host-seeking mosquitoes [7] to determine their biting rate, infection prevalence, and consequently the intensity of malaria transmission they mediate. However, HLC raises ethical concerns because catchers are exposed to vectors that could be potentially infective. It is also labour intensive and unreliable due to variation in attractiveness and skill of the catchers who act as bait hosts [1,8-10]. The continued application of this tool in the surveillance of malaria transmission in sub-Sahara Africa requires careful re-examination and re-justification, with a view to developing and characterizing safer alternative tools that are comparably sensitive.

Over the years, a number of alternative sampling tools that avoid human contact with mosquitoes have been evaluated. These have exhibited wide variations in efficacy and cost, and may not be practical for adoption on programmatic scales in poor malaria-endemic countries $[1,6]$. One of the most commonly employed tools for catching host-seeking malaria vectors in particular is the Centres for Disease Control and Prevention miniature light trap (CDC-LT), which is typically positioned indoors near an occupied net $[11,12]$. Numerous studies have shown the effectiveness of CDC-LTs over a wide range of transmission systems in Africa [12-17]. The positioning of the CDC-LT during sampling influences the sensitivity with which it samples adult female mosquitoes [16] and this trap is almost equally effective when occupants are sleeping under a treated or untreated bed net $[18,19]$.

However, where indoor-targeted insecticidal based interventions such as long-lasting insecticide treated nets (LLINs) and indoor residual spraying (IRS) have drastically reduced endophilic and endophagic vectors [20,21], traps for capturing host-seeking mosquitoes outside of houses are considered more suitable to sample the exophagic vectors that become increasingly important contributors to the residual vector population as intervention coverage is scaled up [4,22-25]. While capture methods primarily targeting host-seeking mosquitoes are ideal for quantifying human exposure to bites and studying host attack behaviours, resting and exit traps are more appropriate for studying resting behaviours and sampling fed mosquitoes to determine the source of blood obtained $[5,6]$.
The characteristic indoor resting (endophilic) behaviour of Anopheles gambiae Giles, An. arabiensis Patton and $A n$. funestus Giles underpins the common use of indoor knockdown pyrethrum spray catches (PSC) and hand collections using a mouth aspirator when surveying [6]. The major drawbacks associated with the hand collection method for resting mosquitoes is poor sensitivity, the laborious nature of rigorous searches through all the irregular surfaces of rural houses, and the great variability in skills and motivation among collectors $[5,26]$. PSC may be expensive to sustain for routine monitoring [26] while the repellence and persistence of the pyrethrum used precludes sampling in the same dwelling more than twice a week [5,27]. Other sampling methods such as, resting boxes (RB), clay pots and bed net traps have been evaluated under different epidemiological settings in Africa with varying degrees of success [28-37]. While window exit traps (WET) have been used for monitoring vector density trends in parts of southern Africa and Bioko island in central Africa [38,39], their efficacy is undoubtedly affected by variations in house design and behavioural patterns of both mosquitoes and humans [40].

A recent review [1] has highlighted the lack of consistency, comparability and characterisation of the numerous, diverse entomological survey tools used to measure malaria transmission. Recent evaluations of a newly developed Ifakara Tent Trap Design C (ITT-C) [41] show that, unlike the $\mathrm{B}$ design that preceded it $[37,42]$, the ITT$\mathrm{C}$ is a genuinely exposure-free tool that probably represents a relatively sensitive and practical mode of sampling malaria vectors for routine surveillance purposes [40], notably through community-based trapping schemes with epidemiological predictive power [43]. Here we report a comparative evaluation of the ITT-C, CDC-LT, RB and WET methods that do not necessitate increased human exposure to mosquito bites, compared to the gold standard HLC which does, in a rural part of Zambia with stable endemic transmission mediated primarily by Anopheles funestus Giles [44,45]. Insecticidal interventions, such as LLINs and IRS can alter survival rates, as well as entry, feeding, resting and exiting behaviours within houses [46], and these two interventions are sometimes combined in parts of Zambia and elsewhere in Africa, with the intention of achieving greater impact than with either alone [47-49]. The influence of supplementing LLINs with IRS upon the efficacy of these trapping methods was, therefore, also assessed by comparing capture rates and sample composition in and immediately outside of houses with both interventions versus those with LLINs alone.

\section{Methods}

Study area

The study was conducted in Chisobe and Nyamumba villages situated between Kasinsa and Chitope rural health 
centres in Luangwa district (Figure 1) which is about $255 \mathrm{~km}$ east of Lusaka. Chisobe and Nyamumba are about $2-3$ kilometres apart. Luangwa is at latitude $-15^{\circ} 41^{\prime} \mathrm{E}$, and longitude $30^{\circ} 08^{\prime} \mathrm{S}$. It is approximately $370 \mathrm{~m}$ above sea level. The wet season runs from November to April and the dry season from June to September with October and May being transitional months. Annual rainfall varies from 600 to $1,400 \mathrm{~mm}$ with mean daytime temperatures ranging from $10^{\circ} \mathrm{C}$ to $44^{\circ} \mathrm{C}$. There are about 26,000 inhabitants in the district who predominantly practice fishing. They also practice animal husbandry and grow seasonal crops.

\section{Study design}

The study was conducted during two intervals chosen within the dry and wet seasons, specifically from September to October 2009 and from February to March 2010, respectively. A $3 \times 3$ Latin Square design was used for the rotated assignment of mosquito sampling methods to experimental units (houses). In each village (Chisobe and Nyamumba), two groups of three houses which were clustered together and identified as distinct experimental blocks with one group comprising those containing LLINs whilst the other also had LLINs but were also treated with IRS. IRS was applied using a deltamethrin formulation (K-Othrine ${ }^{\circledR}$ WG 250, Bayer Environmental Sciences) at a rate of $20 \mathrm{mg}$ of active ingredient per $\mathrm{m}^{2}$ by an experienced spray operator trained at the National Malaria Control Centre.

At the time of the experiment, the only major intervention in the district was the use of PermaNet 2.0 ${ }^{\circledR}$ LLINs (Vestergaard Frandsen SA) distributed through mass distribution campaigns and ante-natal clinics by the Ministry of Health and its partners. As IRS was not an intervention implemented in the district at that time, we therefore purposely sprayed only the selected houses in the LLINs plus IRS blocks to conform to the study design.

Each block was treated as a self-contained trio of numbered (1, 2 and 3) houses in which a Latin Squares rotation sequence was followed throughout the study period. In each of the blocks, the first treatment comprised the HLC conducted both indoors and outdoors and was randomly assigned to one of the numbered houses. The second treatment consisted of a CDC light trap beside an occupied LLIN inside the house, with an ITT-C (Elastic Products Manufacturing Co. Ltd, 67 Bibi Titi Mohamed Road, P.O. Box: 20872, Dar es Salaam, United Republic of Tanzania) placed approximately 5 metres outside of the house, and was assigned to the next highest number. The ITT-C is a canvas tent trap which is about $2000 \mathrm{~mm}$ long, $1000 \mathrm{~mm}$ wide and $1250 \mathrm{~mm}$ high with six funnel-shaped mosquito entrances which enables entry while restricting mosquito exit [41]. Two netting compartments are $700 \mathrm{~mm}$ apart and have sealable cotton sleeves to enable the collection of mosquitoes while avoiding bites. The collecting chambers are further supported with two strings to avoid collapse and further human-mosquito contact. The third treatment consisted of two resting boxes (one placed indoors and the other outdoor) and a window exit trap and was assigned to the next highest number. These collection methods have been described in detail in a similar study conducted in urban Tanzania [40]. Each of the

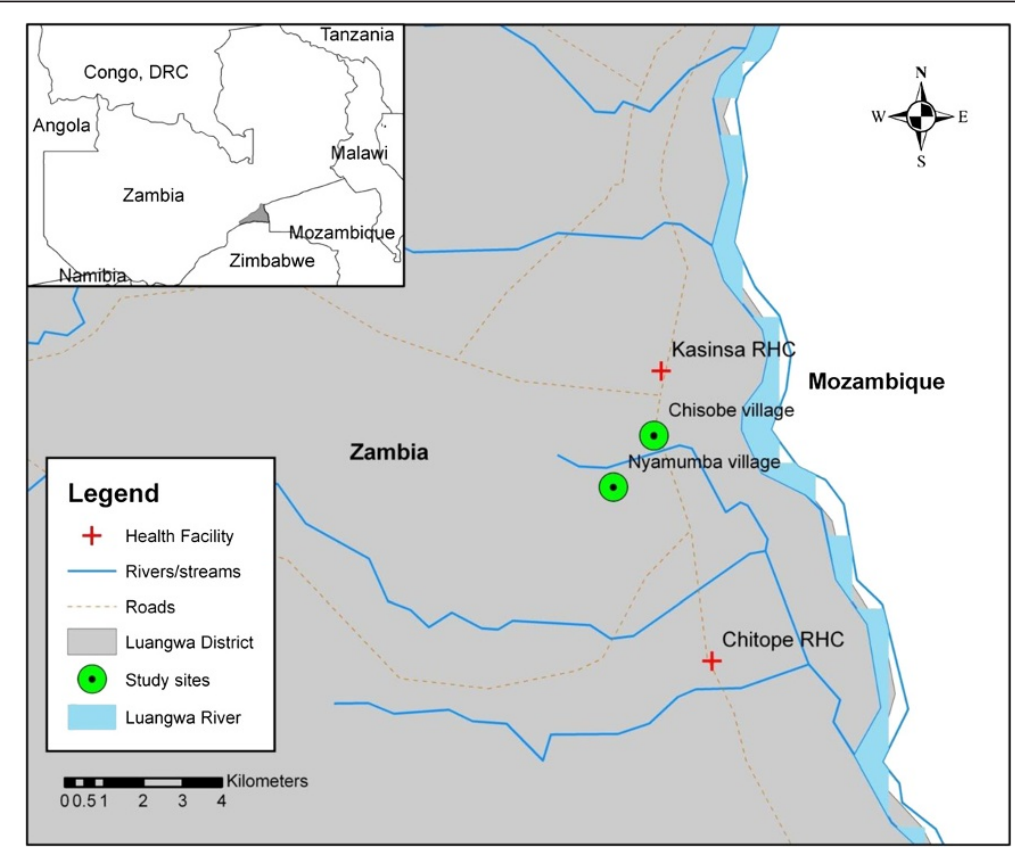

Figure 1 Location of study site (Chisobe and Nyamumba) in Luangwa district. 
three sets of indoor and outdoor collection methods was rotated through the three different houses in increasing order according to their assigned house number, for three consecutive nights in each of 10 rounds, to achieve a balanced data set reflecting an equal number of samples from each treatment-house combination, and time period (rounds). This series of 10 rounds of Latin Squares rotations in 4 blocks over a period of 30 consecutive nights was conducted in both the dry and wet seasons. To compensate for the relative attractiveness of individuals to mosquitoes $[8,9]$ as a confounding factor, the same individual volunteers, who were retained in each house for the duration of the study, were exchanged between indoor (HLC or CDC-LT) and outdoor (HLC or ITT-C) stations each night in a crossover design. For the third treatment, where no human-baited outdoor catches were conducted, both volunteers slept within the house if they were from the same household, otherwise only the volunteer who owned the house and who subsequently conducted HLC indoors and slept under an LLIN when applying CDC-LT occupied the house. In order to ensure comparability, all methods for trapping host-seeking mosquitoes were conducted from 19:00 hrs to 07:00 hrs and all the RBs and WET were emptied at 07:00 hrs after operating for $12 \mathrm{hrs}$ using hand held aspirators as described by Sikulu et al. (2009). Collections from the hourly catches from each catcher conducting HLC were placed in separate cups. Individuals collecting mosquitoes by HLC were allowed to rest for 15 minutes in each hour of collection. Approximately 20 minutes were required to aspirate mosquitoes from each of the ITT-C, CDC-LT, RBs and the WET methods. A team of supervisors conducted random and regular on spot checks to ensure that acceptable standards of execution were maintained by the volunteers.

\section{Mosquito processing}

Mosquitoes were collected from each trap and identified in the field. Female Anopheles mosquitoes were identified to species morphologically [50] and preserved individually in silica gel. Male anophelines were only identified, recorded and discarded. They did not form any part of the analysis. An. gambiae sensu lato and $A n$. funestus sensu lato samples were preserved for circumsporozoite ELISA for infectivity rates [51] and Polymerase Chain Reaction (PCR) for species identification [52,53]. Approximately 83\% (1387) and 11\% (932) of the total specimen morphologically identified as $A n$. gambiae s.l. and An. funestus s.l., respectively were analysed to determine species identity by PCR $[52,53]$ and those which successfully amplified were used for further circumsporozoite ELISA analysis [51]. All identified culicine mosquitoes were recorded as either male or female and discarded.

\section{Data analysis}

All the data were entered using the 2007 Microsoft Excel version. Analysis was performed following the Generalized Linear Mixed Models (GLMM) using R software version 2.15.1. augmented with the Matrix, lattice and lme4 packages. Mixed effects models were used so that fixed effects variables could be used to estimate the effect of factors of interest while accounting for repeated measurements and the influence of other variables such as date and household with many levels as random effects.

\section{Relative abundance, mean catches and sensitivity per sampling method}

The relative catches of the female $A n$. quadriannulatus, An. funestus, and other anopheline and culicine mosquitoes by the different mosquito sampling methods, as compared to the reference method (HLC-indoor), were analysed by fitting GLMMs as follows. The number of catches of the specific mosquito taxon was treated as the dependent variable, to which a Poisson distribution with a logarithm link function was applied. The sampling method, village, treatment (LLINs alone versus LLINs plus IRS) and season were fitted as fixed effects while date (d.f. $=60$ ) and household (d.f. $=12$ ) were treated as random effects. The exponential of the parameter estimates (and 95\% confidence intervals) for each method was calculated to represent the relative rate of catching mosquitoes compared to the standard reference method (HLC indoor). We calculated the mean by fitting GLMM with the sampling method treated as a categorical factor and both date and house as random effects using a Poisson distribution with logarithm link function and determined as described above. Similarly, we used the outputs from GLMM model to test for and quantify the effect of treatment, season and village on the abundance of mosquitoes of different taxa.

\section{Influence of indoor residual spraying upon the numbers of human-feeding An. funestus caught by all sampling methods}

In order to analyse the effect of treating a house with IRS upon house entry and feeding on humans by mosquitoes, we fitted GLMMs with Poisson distribution, treating the number of mosquitoes caught with each trapping method in each house and station (in versus out) as the dependent variable and IRS treatment status, village and season as fixed effects. Household (d.f. =12) and date (d.f. $=60)$ were treated as random effects.

Influence of sampling method on the proportion of all An. quadriannulatus and An. funestus caught which were fed In order to analyse the effect of trapping method upon the proportion of mosquitoes which had fed, we applied binomial logistic regression by fitting a GLMM with a 
logit link function for the proportion of fed female mosquitoes caught by each method, defined by the total number of fed mosquitoes as the numerator and the total catch of all female mosquitoes of all physiological status as the denominator. Abdominal status was classified as either fed (partly fed and fed) or unfed (unfed, partly gravid and fully gravid) and the fixed effects included village, season, and IRS treatment status while date and household were included as random effects.

\section{Ethical considerations}

The study protocol was granted approval by The National Ethics Committee based at The University of Zambia (IRB00001131 of I0RG0000774) and the Ethical Review Board of the Liverpool School of Tropical Medicine (09.60). All individuals in the study had consented to participate in the study following a thorough description of the benefits and risks involved. Consenting participants were administered with Deltaprim ${ }^{\odot}$ drug (one of the recommended drugs for chemoprophylaxis in Zambia) as prophylaxis every week.

\section{Results}

\section{Mean catches and relative sensitivities of alternative} sampling methods in relation to indoor

\section{HLC}

Summary of total catches, mean catches per trap night and relative sensitivities of alternative sampling methods in relation to HLC indoor are indicated in Table 1. A total of 19664 female mosquitoes were caught in 60 sampling nights, with 7.4\% comprising An. gambiae s.l., 38.9\% An. funestus s.l., $22.6 \%$ other anophelines and $31.1 \%$ culicine mosquitoes. The other anophelines constituted mainly $A n$. coustani, An. pretoriensis, An. squamosus and An. rufipes. Out of the 932 (11\%) specimens of An. funestus s.l., tested by PCR, only $47 \%(n=440)$ successfully amplified. Most were identified as An. funestus sensu stricto $(72.2 \%, \mathrm{n}=317)$ with the remainder being $A n$. rivulorum $(16.2 \%, \mathrm{n}=71)$, An. parensis $(9.8 \%, \mathrm{n}=43)$, An. vaneedeni $(1.4 \%, \mathrm{n}=7)$ and An. lessoni $(0.5 \%, \mathrm{n}=2)$. From the total of 1387 (83\%) An. gambiae s.l. specimens tested by PCR, 1169 (85\%) successfully-amplified. The vast majority were $A n$. quadriannulatus $(95.2 \%, \mathrm{n}=1112)$ with only a very small number of An. arabiensis $(3.9 \%, \mathrm{n}=46)$ and An. gambiae sensu stricto $(0.9 \%, \mathrm{n}=11)$. In subsequent analysis, we therefore report results for the Anopheles funestus group and the An. gambiae complex as approximately representing Anopheles funestus s.s. and An. quadriannulatus, respectively. Anopheles rivulorum (18.3\%, $\mathrm{n}=13)$ and $A n$. funestus s.s $(2.2 \%, \mathrm{n}=7)$ were the only species from the An. funestus group, or any other Anopheles taxon, found to be infected with $P$. falciparum sporozoites. However, none of these specimens were re-tested following heating of the homogenates, so the possibility of exaggerated sporozoite prevalence due to false positives, for $A n$. rivulorum in particular, cannot be excluded [54].

Statistical estimates of the magnitude and significance of differences in relative rates at which each trapping method captured mosquitoes are presented in Table 1. Of all the alternative methods, only CDC-LT performed better than HLC indoor for sampling both An. quadriannulatus and $A n$. funestus, being over one and a half times more sensitive for both species. For An. funestus, ITT-C placed outdoors exhibited over three fourths the sensitivity of HLC and may therefore be useful for trapping this malaria vector species. However, for An. quadriannulatus, other anophelines and culicines, indoor CDC-LT proved the only reasonably sensitive alternative to HLC. For culicines, indoor CDC-LT exhibited more than three fourths the sensitivity of HLC which yielded approximately equal catches indoors and outdoors. While the ITT-C was the only alternative method other than CDC-LT that caught any useful numbers of culicines, it exhibited quite low sensitivity and might have limited utility for this important taxon that transmits a wide range of parasites and viruses of public health importance. ITT-C also exhibited extremely poor sensitivity for An. quadriannulatus and other anophelines. However, the RBs and the WET sampled much lower catches for all the mosquito taxa. Mosquitoes were observed on several occasions escaping from the RBs placed outdoors at sun rise prior to collection time.

\section{Influence of indoor residual spraying on the catches of An. funestus by all sampling methods}

Supplementation of LLINs with IRS had no influence on the catches of An. funestus by indoor HLC $(P=0.270)$, outdoor HLC $(P=0.242)$ and CDC-LT $(P=0.229)$ placed indoors. While IRS appeared to increase catches in ITTC placed outdoors (RR [95\%CI] $=1.399$ [1.016, 1.929], $P$ $=0.040$ ), this apparent effect is most likely spurious, arising from the relatively small number of houses assigned to each treatment.

\section{Influence of sampling method on the proportion of all} fed An. quadriannulatus and An. funestus captured

All specimens of $A n$. quadriannulatus caught with RBs placed outdoors had previously fed. HLCs, CDC-LT and ITT-C each collected less than a third of the fed $A n$. quadriannulatus while RBs placed indoors and WETs caught none. However, RBs placed both indoors and outdoors collected high proportions of fed An. funestus. Approximately over a third of An. funestus mosquitoes caught by HLC and WET had fed. The former is a remarkably high proportion for a sample of host-seeking vectors and it is reassuring that this proportion is reduced in samples from both ITT-C and CDC-LT that 
Table 1 Number of mosquitoes caught by different sampling methods for $\mathbf{2 4 0}$ trap nights each and their relative rates in reference to the indoor human landing catches, as determined by fitting generalized linear mixed models ${ }^{a}$

\begin{tabular}{|c|c|c|c|c|}
\hline \multirow[t]{2}{*}{ Sampling method } & \multicolumn{2}{|c|}{ Catch $^{\text {b }}$} & \multicolumn{2}{|c|}{ Relative sensitivity $^{c}$} \\
\hline & Total & Mean $[95 \% \mathrm{Cl}]$ & $\operatorname{RR}[95 \% \mathrm{Cl}]$ & $P$ value \\
\hline \multicolumn{5}{|l|}{ Anopheles quadriannulatus } \\
\hline HLC indoor & 405 & $1.687[1.531,1.860]$ & $1.00^{d}$ & $N A^{e}$ \\
\hline HLC outdoor & 242 & $1.004[0.885,1.139]$ & $0.597[0.509,0.700]$ & $<0.001$ \\
\hline CDC light trap & 784 & $3.267[3.046,3.504]$ & $1.873[1.653,2.122]$ & 0.997 \\
\hline Ifakara tent trap - C & 21 & $0.088[0.057,0.134]$ & $0.050[0.032,0.078]$ & $<0.001$ \\
\hline Window exit trap & 1 & $0.004[0.001,0.030]$ & $0.002[0.000,0.015]$ & $<0.001$ \\
\hline Resting boxes indoor & 0 & $N E^{f}$ & $N E^{f}$ & $N E^{f}$ \\
\hline Resting boxes outdoor & 2 & $0.008[0.002,0.033]$ & $0.004[0.001,0.016]$ & $<0.001$ \\
\hline \multicolumn{5}{|l|}{ Anopheles funestus } \\
\hline $\mathrm{HLC}$ indoor & 1749 & $7.287[6.954,7.637]$ & $1.00^{d}$ & $N A^{e}$ \\
\hline HLC outdoor & 1635 & $6.784[6.463,7.121]$ & $0.928[0.868,0.993]$ & $<0.001$ \\
\hline CDC light trap & 2630 & $10.958[10.547,11.385]$ & $1.532[1.441,1.628]$ & $<0.001$ \\
\hline Ifakara tent trap - C & 1410 & $5.875[5.576,6.190]$ & $0.821[0.765,0.881]$ & $<0.001$ \\
\hline Window exit trap & 71 & $0.296[0.234,0.373]$ & $0.040[0.032,0.051]$ & $<0.001$ \\
\hline Resting boxes indoor & 38 & $0.158[0.115,0.218]$ & $0.022[0.016,0.030]$ & $<0.001$ \\
\hline Resting boxes outdoor & 110 & $0.458[0.380,0.553]$ & $0.063[0.052,0.076]$ & $<0.001$ \\
\hline \multicolumn{5}{|l|}{ Other anophelines } \\
\hline $\mathrm{HLC}$ indoor & 1661 & $8.046[7.695,8.413]$ & $1.00^{d}$ & $N A^{e}$ \\
\hline HLC outdoor & 2064 & $9.685[9.300,10.086]$ & $1.207[1.137,1.287]$ & $<0.001$ \\
\hline CDC light trap & 661 & $2.754[2.552,2.972]$ & $0.337[0.308,0.369]$ & $<0.001$ \\
\hline Ifakara tent trap - C & 28 & $0.117[0.081,0.169]$ & $0.014[0.010,0.021]$ & $<0.001$ \\
\hline Window exit trap & 7 & $0.029[0.014,0.061]$ & $0.003[0.002,0.007]$ & $<0.001$ \\
\hline Resting boxes indoor & 4 & $0.017[0.006,0.044]$ & $0.002[0.001,0.005]$ & $<0.001$ \\
\hline Resting boxes outdoor & 20 & $0.083[0.054,0.129]$ & $0.010[0.006,0.015]$ & $<0.001$ \\
\hline \multicolumn{5}{|l|}{ Culicine species } \\
\hline HLC indoor & 1971 & $8.296[7.939,8.668]$ & $1.00^{d}$ & $N A^{e}$ \\
\hline HLC outdoor & 1921 & $8.033[7.683,8.399]$ & $0.971[0.912,1.0349]$ & 0.349 \\
\hline CDC light trap & 1782 & $7.425[7.088,7.778]$ & $0.871[0.817,0.930]$ & $<0.001$ \\
\hline Ifakara tent trap - C & 369 & $1.538[1.388,1.703]$ & $0.180[0.161,0.202]$ & $<0.001$ \\
\hline Window exit trap & 54 & $0.225[0.172,0.294]$ & $0.025[0.019,0.033]$ & $<0.001$ \\
\hline Resting boxes indoor & 6 & $0.025[0.011,0.056]$ & $0.003[0.001,0.006]$ & $<0.001$ \\
\hline Resting boxes outdoor & 18 & $0.075[0.047,0.119]$ & $0.008[0.005,0.013]$ & $<0.001$ \\
\hline \multicolumn{5}{|c|}{$\begin{array}{l}\text { As described in the methods section, village, season and treatment were all included as fixed effects while } \\
\text { In sampling An. quadriannulatus, both village and treatment did not significantly affect }(P=0.894 \text { and } 0.0845 \\
\text { methods. The catches of An. funestus were also significantly affected by village }(P=0.004) \text { and treatment }(p= \\
\text { culicines were not significantly affected by village }(P=0.268 \text { and } 0.265) \text { and treatment }(P=0.717 \text { and } 0.721) \\
\text { were significantly affected by season }(P<0.001) \text {. } \\
\text { b Mean and } 95 \% \text { confidence intervals (Cl) were estimated by fitting generalised linear mixed models as descr } \\
\text { house were included in a model without intercept. } \\
\text { c Sensitivity of the sampling method catch with reference to HLC placed indoors (RR indicate Relative Rate). } \\
\text { d Reference method. } \\
\text { e Not applicable. } \\
\text { f Not estimable due to no mosquito catch. }\end{array}$} \\
\hline
\end{tabular}

are assumed to protect the human participant from exposure to the collected mosquitoes (Table 2).

\section{Discussion}

Amongst the methods that capture host-seeking mosquitoes, the CDC-LT placed near an occupied net compares well with HLC. This observation is consistent with many reports from elsewhere in the tropics in sampling various pathogencarrying mosquito species $[14,15,17,31,35,40,42,55-60]$ except for an evaluation in Dar es Salaam, Tanzania which showed very poor sensitivity of CDC-LT in this urban environment. While previous studies were limited to $A n$. 
Table 2 Influence of sampling method on the proportion of fed An. quadriannulatus and An. funestus which were captured $^{\mathrm{a}}$

\begin{tabular}{|c|c|c|c|}
\hline Sampling method & Percentage (Proportion fed) & $\mathrm{OR}^{\mathrm{b}}[95 \% \mathrm{C.I}]$ & $P$ value \\
\hline \multicolumn{4}{|c|}{ Anopheles quadriannulatus } \\
\hline HLC indoor & $24.4(99 / 405)$ & $1.00^{c}$ & $N A^{d}$ \\
\hline HLC outdoor & $29.3(71 / 242)$ & $1.900[1.253,2.881]$ & 0.003 \\
\hline CDC light trap & $12.9(101 / 784)$ & $0.417[0.292,0.596]$ & $<0.001$ \\
\hline Ifakara tent trap $-\mathrm{C}$ & $28.6(6 / 21)$ & $1.251[0.430,3.642]$ & 0.682 \\
\hline Window exit traps & $(0 / 1)$ & $N E^{e}$ & $N E^{e}$ \\
\hline Resting boxes indoor & $(0 / 0)$ & $N E^{e}$ & $N E^{e}$ \\
\hline Resting boxes outdoor & $100(2 / 2)$ & $N E^{e}$ & $N E^{e}$ \\
\hline \multicolumn{4}{|l|}{ Anopheles funestus } \\
\hline HLC indoor & $34.8(608 / 1749)$ & $1.00^{c}$ & $N A^{d}$ \\
\hline HLC outdoor & $37.2(608 / 1635)$ & $1.188[1.017,1.387]$ & 0.030 \\
\hline CDC light trap & $20.6(541 / 2630)$ & $0.543[0.467,0.633]$ & $<0.001$ \\
\hline Ifakara tent trap $-\mathrm{C}$ & $14.1(199 / 1410)$ & $0.261[0.215,0.317]$ & $<0.001$ \\
\hline Window exit trap & $38.0(27 / 71)$ & $1.086[0.643,1.835]$ & 0.758 \\
\hline Resting boxes indoor & $73.7(28 / 38)$ & $4.486[2.059,9.776]$ & $<0.001$ \\
\hline Resting boxes outdoor & $72.7(80 / 110)$ & $5.899[3.688,9.434]$ & $<0.001$ \\
\hline
\end{tabular}

gambiae s.s. and An. arabiensis in Tanzania [40-42], this is the first report showing that ITT-C appears to be a useful option for sampling host-seeking An. funestus in an external trial site in Zambia. This species is among the most important malaria vectors in Africa generally and Zambia specifically, and it is notable that the ITT-C sampled considerably more An. funestus than any other mosquito taxon in this study. This is particularly noteworthy because ITT-C is the only sampling tool that has yet been successfully applied through quality assured communitybased trapping schemes with epidemiological predictive power as a malaria risk indicator [43]. ITT-C might, therefore, be applicable as an option for programmatic use across much of Africa where An. funestus is an important vector of malaria $[61,62]$. Nevertheless, the poor sensitivity ITT-C exhibited for culicines, An quadriannulatus and other anophilines suggests caution, and that it requires evaluation across a broader diversity of contexts before it can be recommended for wide spread use. Indeed it has recently been emphasised that there is a great need to consistently compare sampling methods across diverse transmission patterns in Africa and that such comparative evaluations are conspicuous by their absence from the literature [1]. Critically, this study used a very similar design to that previously implemented in Dar es Salaam, so that the two evaluations from two very different contexts can be directly compared.
The observation by Govella et al. [40] that houses have many, highly variable entry and exit points, was also noted in our study area and might well explain the very low sensitivity of WET. The poor sensitivity of RBs is most likely explained by the fact that they represent too small a proportion of the total suitable resting surface area available to mosquitoes indoors and especially outdoors. Outdoor resting tools are also prone to natural mosquito predators which may contribute to the low catches [63] and mosquitoes also tend to leave when illumination increases as sunrise approaches. While other reports describe useful sensitivity levels of boxes [26,36] and pots [34] as resting traps, our observation that both the RB and WET methods exhibited poor sensitivities for sampling all mosquito taxa are consistent with some previous reports from neighbouring Tanzania [37,40]. Much of the dramatic drop in capture efficacy reported by these recent studies in Zambia (Table 1) and Tanzania [40], relative to previous reports from Kenya [26,34] and Tanzania [36] may well be explained by the presence and coverage levels of insecticidal nets. Given that insecticide-treated nets are estimated to prevent an average of 93\% of exposure for people sleeping under them [48], it is inevitable that this study and a similar recent one in Tanzania in which all occupants used nets [40] both report far lower catches in resting traps than host-seeking traps because only a small minority of host-seeking mosquitoes will 
successfully survive, acquire a blood meal and consequently rest in the same house they entered.

However, this cannot entirely explain the comparatively low numbers of mosquitoes caught with resting boxes ( $\leq 1 \%$ sensitivity relative to HLC for all taxa except An. funestus). In the case of the WET, any deterred mosquitoes are readily available for capture upon exit, as demonstrated by recent trials of completely intact nets in experimental huts combining baffled entry points with comprehensive exit trapping of all remaining eaves and windows [64,65]. Fundamental limitations of sampling sensitivity of these RBs and WET formats are therefore probably important so more sensitive approaches such as PSC [5] and backpack aspirators [66] should be evaluated in a similarly standardised way. While these resting traps may be useful for some applications in some settings, the inferring quantitative levels of human exposure based on absolute numbers of mosquitoes caught may not be reliably recommended or readily interpreted in a standardized way. However, it is crucial to consider whether the focus of a given entomological survey is to quantify human exposure, understand vector resting behaviour, or identify blood meal sources of fed mosquitoes when selecting appropriate sampling tools [5]. Therefore sensitivity may not be the most important criterion in many cases.

In our study site, An. quadriannulatus appears to be the predominant species amongst the An. gambiae complex and was caught more indoors than outdoors by the CDCLT and HLC methods. While these results seem unexpected because An. quadriannulatus is usually associated with outdoor biting and a preference for non-human hosts [31,50], it does occasionally bite people $[67,68]$ but is thought to contribute negligibly to malaria transmission $[67,68]$. Torr and colleagues (2008) showed that, when humans are indoors, their odour attracts more zoophilic species than those stationed outdoors and this may partially explain the results obtained in this study [68]. The high numbers of An. quadriannulatus caught indoors here may also result from the fact that, apart from the catcher, other household inhabitants were present but covered with nets inside these homes, whereas the human single baits collected outdoors were alone. While the preference of An. funestus for feeding indoors was statistically significant (Table 1), it was quantitatively very small and of little biological significance, as appears to be the case for most malaria vector populations in Africa [69]. The vast majority of human exposure occurs indoors in this setting, and elsewhere in Africa [69] simply because the peak hours of An. funestus biting activity coincide with almost all humans going into their houses to sleep [63].

Although IRS treatment of houses which already had LLINs appeared to have no impact on the catches of $A n$. funestus across all trap types, it appeared to increase catches by ITT-C placed outdoors. This presumably spurious result probably arises from the small number of houses assigned to each treatment because it is inconsistent with results reported here for the gold-standard HLC method and reported previously using logistic models of the proportion of mosquitoes caught indoors rather than outdoors at a given house [63]. So overall, it is notable that IRS with deltamethrin had so little apparent impact on house entry and subsequent host attack rates. This observation is consistent with a number of recent experimental hut evaluations $[64,70,71]$ of modern pyrethroid formulations, confirming that this intervention format provides little direct protection to individual households and acts exclusively through community-level suppression of vector populations and malaria transmission.

It is worth noting that whilst large numbers of both An. quadriannulatus and An. funestus s.l. were caught indoors by the CDC-LT and HLC, the majority that had fed were sampled by the sampling methods placed outdoors. It is disconcerting that 24 to $37 \%$ of mosquitoes caught by the HLC methods, especially An. funestus, were blood fed. Presumably most of these either partially fed elsewhere before landing on the human bait to complete the blood meal, or obtained the blood meal from the human bait conducting the HLC. This supports the efforts to search for safer alternatives because these findings suggest that the catchers may have lacked concentration due to exhaustion and were therefore bitten extensively. High proportions of fed mosquitoes were also sampled in the RBs indoors and outdoors because these represent artificial resting places for mosquitoes, which rest most during the gestation phase of their life cycle. The lower proportions of fed An. funestus that were sampled by the ITT-C and fed An. quadriannulatus that were sampled by CDC-LT (Table 2) suggest that these methods do protect the human participants acting as bait and confirm the findings of Govella et al. in an urban Tanzanian setting [40]. It is possible that the substantial proportions of fed An. funestus and An. quadriannulatus in the ITT-C could have used the tent trap as an alternative resting place after feeding elsewhere or were simply attracted to the host for further feeding after being partially fed elsewhere. However, we could not substantiate this because our study did not include host blood meal analysis.

Despite these ambiguities and study limitations, these experiments do demonstrate the importance of evaluating the efficacy of alternative exposure-free sampling tools for routine monitoring of malaria transmission, in comparison with each other and with gold standard HLC in different settings. It further highlights the need to specifically evaluate sampling methods based on their ability to selectively trap either host-seeking, exiting, or resting mosquitoes, and to capture them with sufficient sensitivity relative to absolute house entry and host attack rates within houses. 


\section{Conclusions}

Although CDC-LT seems to be the most sensitive option for trapping host-seeking mosquitoes in this setting, the continuous need to recharge batteries might be challenging for surveillance systems in rural communities, particularly where electricity is not readily available. This may pose particular challenges for routine programmatic monitoring applications outside of research studies, notably community-based trapping schemes with little supervision and only occasional quality assurance [43]. The ITT-C appears to offer a reasonable alternative that does not depend on electrical power. However, its bulkiness could be a significant disadvantage that may limit its application in routine malaria surveillance systems, especially community-based schemes with little or no motorized transport. While RBs collect high proportions of fed mosquitoes, they have very low relative sensitivity in comparison with host-seeking methods, so similarly standardized evaluation of more promising methods for capturing resting mosquitoes, such as mechanized aspirators [66] and pyrethrum spray catch [5] should be considered. The efficacy of neither CDC-LT nor ITT-C appears to be affected by the application of pyrethroid-based IRS to houses already containing LLINs.

\section{Competing interests}

Authors declare that they have no competing interests.

\begin{abstract}
Authors' contributions
CHS, AS and GFK designed the study collected data analysed and drafted the manuscript. TLR: Assisted at the inception of the study through data entry and field design operations. JC and DC: Collected field data and morphologically identified all the mosquitoes in the field. JM: Provided the study map and assisted in the data analysis AS: Together with CHS and GFK designed the study and oversaw the field implementation of the study and contributed to the formulation of the hypothesis and drafting the manuscript. GFK: Together with AS and CHS designed the study and conceived the study hypotheses' and contributed in drafting the manuscript. All authors read and approved the final version of the manuscript.
\end{abstract}

\section{Acknowledgements}

We thank the Ministry of Health, National Malaria Control Centre, Luangwa District Health Office and communities in Chisobe and Nyamumba for their co-operation and support. We thank Dr Nicodem Govella for arranging supply of ITT-C and for his advice on how to use them. Financial support was provided by the Bill \& Melinda Gates Foundation through the Malaria Transmission Consortium (Award number 45114) coordinated by Dr Neil Lobo and Prof Frank Collins at Notre Dame University.

\footnotetext{
Author details

${ }^{1}$ National Malaria Control Centre, Chainama Hospital College Grounds, Off Great East road, P.O. Box 32509, Lusaka, Zambia. ${ }^{2}$ Vector Biology Department, Liverpool School of Tropical Medicine, Pembroke Place, Liverpool L3 5QA, UK. ${ }^{3}$ Ifakara Health Institute, Biomedical \& Environmental Thematic Group, P. O. Box 53, Ifakara, Morogoro, United Republic of Tanzania. ${ }^{4}$ Malaria Control, Evaluation and Partnership in Africa (MACEPA), Chainama Hospital College Grounds, Off Great East road, P.O. Box 32509, Lusaka, Zambia. ${ }^{5}$ James Cook University, School of Public Health, Tropical Medicine and Rehabilitation Sciences, Cairns 4870, Australia.
}

\section{References}

1. Kelly-Hope LA, McKenzie FE: The multiplicity of malaria transmission: a review of entomological inoculation rate measurements and methods across sub-Saharan Africa. Malaria J 2009, 8:19.

2. Bugoro H, Cooper RD, Butafa C, Iro'ofa C, Mackenzie DO, Chen CC, Russell $\mathrm{TL}$ : Bionomics of the malaria vector Anopheles farauti in Temotu Province, Solomon Islands: issues for malaria elimination. Malaria J 2011, 10:133.

3. Meyrowitsch DW, Pedersen EM, Alifrangis M, Scheike TH, Malecela MN, Magesa SM, Derua YA, Rwegoshora RT, Michael E, Simonsen PE: Is the current decline in malaria burden in sub-Saharan Africa due to a decrease in vector population? Malaria J 2011, 10:188.

4. Russell TL, Govella NJ, Azizi S, Drakeley CJ, Kachur SP, Killeen GF: Increased proportions of outdoor feeding among residual malaria vector populations following increased use of insecticide-treated nets in rural Tanzania. Malaria J 2011, 10:80.

5. Service MW: Field Sampling Methods. London and New York: Elsevier Applied Science; 1993

6. Service MW, Townson H: The Anopheles vector. New York: Arnold; 2002

7. Service MW: A critical review of procedures for sampling populations of adult mosquitoes. Bull Entomol Res 1977, 67:343-382.

8. Lindsay SW, Adiamah JH, Miller JE, Pleass RJ, Armstrong JR: Variation in attractiveness of human subjects to malaria mosquitoes (Diptera: Culicidae) in The Gambia. J Med Entomol 1993, 30:368-373.

9. Knols BG, de Jong R, Takken W: Differential attractiveness of isolated humans to mosquitoes in Tanzania. Trans R Soc Trop Med Hyg 1995, 89:604-606.

10. Mukabana WR, Takken W, Coe R, Knols BG: Host-specific cues cause differential attractiveness of Kenyan men to the African malaria vector Anopheles gambiae. Malaria J 2002, 1:17.

11. Garrett-Jones C, Magayuka SA: Studies on the natural incidence of Plasmodium and Wuchereria infections in Anopheles in rural East Africa:1 Assessment of density by trapping hungry female Anopheles gambiae Giles; species A. Geneva: World Health Organization; 1975.

12. Sudia WD: Battery operated light-trap, an improved model. Mosq News 1962, 22:126-129.

13. Davis JR, Hall T, Chee EM, Majala A, Minjas J, Shiff CJ: Comparison of sampling anopheline mosquitoes by light-trap and human-bait collections indoors at Bagamoyo, Tanzania. Med Vet Entomol 1995, 9:249-255.

14. Odetoyinbo JA: Preliminary investigation on the use of a light-trap for sampling malaria vectors in the Gambia. Bull World Health Organ 1969, 40:547-560.

15. Mbogo CN, Glass GE, Forster D, Kabiru EW, Githure Jl, Ouma JH, Beier JC Evaluation of light traps for sampling anopheline mosquitoes in Kilifi, Kenya. J Am Mosq Control Assoc 1993, 9:260-263.

16. Mboera LE, Kihonda J, Braks MA, Knols BG: Short report: Influence of centers for disease control light trap position, relative to a human-baited bed net, on catches of Anopheles gambiae and Culex quinquefasciatus in Tanzania. AmJTrop Med Hyg 1998, 59:595-596.

17. Shiff CJ, Minjas JN, Hall T, Hunt RH, Lyimo S, Davis JR: Malaria infection potential of anopheline mosquitoes sampled by light trapping indoors in coastal Tanzanian villages. Med Vet Entomol 1995, 9:256-262.

18. Killeen GF, Tami A, Kihonda J, Okumu FO, Kotas ME, Grundmann H, Kasigudi $\mathrm{N}$, Ngonyani H, Mayagaya $V$, Nathan $R$, et al: Cost-sharing strategies combining targeted public subsidies with private-sector delivery achieve high bednet coverage and reduced malaria transmission in Kilombero Valley, southern Tanzania. BMC Infect Dis 2007, 7:121.

19. Magbity EB, Lines JD: Spatial and temporal distribution of Anopheles gambiae s.l. (Diptera: Culicidae) in two Tanzanian villages: implication for designing mosquito sampling routines. Bull Entomol Res 2002, 92:483-488.

20. Griffin JT, Hollingsworth TD, Okell LC, Churcher TS, White M, Hinsley W, Bousema T, Drakeley CJ, Ferguson NM, Basanez MG, et al: Reducing Plasmodium falciparum malaria transmission in Africa: a model-based evaluation of intervention strategies. PLoS Med 2010, 7:e1000324.

21. Kiware SS, Chitnis N, Devine GJ, Moore SJ, Majambere S, Killeen GF: Biologically meaningful coverage indicators for eliminating malaria transmission. Biol Lett 2012, 8:874-877.

22. Molineaux L, Shidrawi GR, Clarke JL, Boulzaguet R, Ashkar T, Dietz K: The impact of propoxur on Anopheles gambiae s.1. and some other anopheline populations, and its relationship with some pre-spraying variables. Bull World Health Organ 1976, 54:379-389. 
23. Bugoro H, Iro'ofa C, Mackenzie DO, Apairamo A, Hevalao W, Corcoran S, Bobogare A, Beebe NW, Russell TL, Chen CC, et al: Changes in vector species composition and current vector biology and behaviour will favour malaria elimination in Santa Isabel Province. Solomon Islands. Malaria J 2011, 10:287.

24. Mutuku FM, King CH, Mungai P, Mbogo C, Mwangangi J, Muchiri EM, Walker ED, Kitron U: Impact of insecticide-treated bed nets on malaria transmission indices on the south coast of Kenya. Malaria J 2011, 10:356

25. Reddy MR, Overgaard HJ, Abaga S, Reddy VP, Caccone A, Kiszewski AE, Slotman MA: Outdoor host seeking behaviour of Anopheles gambiae mosquitoes following initiation of malaria vector control on Bioko Island, Equatorial Guinea. Malaria J 2011, 10:184.

26. Harbison JE, Mathenge EM, Misiani GO, Mukabana WR, Day JF: A simple method for sampling indoor-resting malaria mosquitoes Anopheles gambiae and Anopheles funestus (Diptera: Culicidae) in Africa. J Med Entomol 2006, 43:473-479.

27. Chareonviriyaphap T, Roberts DR, Andre RG, Harlan HJ, Manguin S, Bangs MJ: Pesticide avoidance behavior in Anopheles albimanus, a malaria vector in the Americas. J Am Mosa Control Assoc 1997, 13:171-183.

28. Edman J, Kittayapong P, Linthicum K, Scott T: Attractant resting boxes for rapid collection and surveillance of Aedes aegypti (L.) inside houses. J Am Mosq Control Assoc 1997, 13:24-27.

29. Kittayapong P, Linthicum KJ, Edman JD, Scott TW: Further evaluation of indoor residual resting boxes for Aedes aegypti surveillance. Deng Bull 1997, 21:77-83.

30. Yasuno M, Rajagopalan PK, Kazmi SJ, LaBreeque GC: Seasonal change in larval habitats and population density of Culex fatigus in Delhi villages. Indian J Med Res 1997, 65:52-64.

31. Laganier R, Randimby FM, Rajaonarivelo V, Robert V: Is the Mbita trap a reliable tool for evaluating the density of anopheline vectors in the highlands of Madagascar? Malaria J 2003, 2:42.

32. Mathenge EM, Misiani GO, Oulo DO, Irungu LW, Ndegwa PN, Smith TA, Killeen GF, Knols BG: Comparative performance of the Mbita trap, CDC light trap and the human landing catch in the sampling of Anopheles arabiensis, An. funestus and culicine species in a rice irrigation in western Kenya. Malaria J 2005, 4:7.

33. Mathenge EM, Omweri GO, Irungu LW, Ndegwa PN, Walczak E, Smith TA, Killeen GF, Knols BG: Comparative field evaluation of the Mbita trap, the Centers for Disease Control light trap, and the human landing catch for sampling of malaria vectors in western Kenya. AmJTrop Med Hyg 2004, 70:33-37.

34. Odiere M, Bayoh MN, Gimnig J, Vulule J, Irungu L, Walker E: Sampling outdoor, resting Anopheles gambiae and other mosquitoes (Diptera: Culicidae) in western Kenya with clay pots. J Med Entomol 2007, 44:14-22.

35. Okumu FO, Kotas ME, Kihonda J, Mathenge E, Killeen GF, Moore SJ: Comparative evaluation of methods used for sampling malaria vectors in the Kilombero valley, south eastern Tanzania. Open Trop Med J 2008, 1:51-55

36. Kweka EJ, Mwang'onde BJ, Kimaro E, Msangi S, Massenga CP, Mahande AM: A resting box for outdoor sampling of adult Anopheles arabiensis in rice irrigation schemes of lower Moshi, northern Tanzania. Malaria J 2009, 8:82.

37. Sikulu M, Govella NJ, Ogoma SB, Mpangile J, Kambi SH, Kannady K, Chaki PC, Mukabana WR, Killeen GF: Comparative evaluation of the Ifakara tent trap- $B$, the standardized resting boxes and the human landing catch for sampling malaria vectors and other mosquitoes in urban Dar es Salaam, Tanzania. Malaria J 2009, 8:197.

38. Mouatcho JC, Hargreaves K, Koekemoer LL, Brooke BD, Oliver SV, Hunt RH, Coetzee M: Indoor collections of the Anopheles funestus group (Diptera: Culicidae) in sprayed houses in northern KwaZulu-Natal, South Africa. Malaria J 2007, 6:30.

39. Sharp BL, Ridl FC, Govender D, Kuklinski J, Kleinschmidt I: Malaria vector control by indoor residual insecticide spraying on the tropical island of Bioko, Equatorial Guinea. Malaria J 2007, 6:52.

40. Govella NJ, Chaki PP, Mpangile JM, Killeen GF: Monitoring mosquitoes in urban Dar es Salaam: evaluation of resting boxes, window exit traps, CDC light traps, Ifakara tent traps and human landing catches. Parasit Vectors 2011, 4:40.

41. Govella NJ, Moore JD, Killeen GF: An exposure-free tool for monitoring adult malaria mosquito populations. AmJTrop Med Hyg 2010, 83:596-600.

42. Govella NJ, Chaki PP, Geissbuhler Y, Kannady K, Okumu F, Charlwood JD, Anderson RA, Killeen GF: A new tent trap for sampling exophagic and endophagic members of the Anopheles gambiae complex. Malaria J 2009, 8:157.

43. Chaki PP, Mlacha YP, Msellem D, Muhili A, Malishee AD, Mtema ZJ, Kiware SS, Zhou Y, Lobo NF, Russell TL, et al: An affordable, quality-assured community-based system for high-resolution entomological surveillance of vector mosquitoes that reflects human malaria infection risk patterns. Malaria J 2012, 11:172.

44. Keating J, Miller JM, Bennett A, Moonga HB, Eisele TP: Plasmodium falciparum parasite infection prevalence from a household survey in Zambia using microscopy and a rapid diagnostic test: implications for monitoring and evaluation. Acta Trop 2009, 112:277-282.

45. Ministry of Health: Zambia National Indicator Survey. Lusaka: National Malaria Control Centre; 2010.

46. Pates $\mathrm{H}$, Curtis C: Mosquito behavior and vector control. Annu Rev Entomol 2005, 50:53-70.

47. Kleinschmidt I, Schwabe C, Shiva M, Segura JL, Sima V, Mabunda SJ, Coleman M: Combining indoor residual spraying and insecticide-treated net interventions. AmJTrop Med Hyg 2009, 81:519-524.

48. Okumu FO, Moore SJ: Combining indoor residual spraying and insecticidetreated nets for malaria control in Africa: a review of possible outcomes and an outline of suggestions for the future. Malaria J 2011, 10:208.

49. Chanda E, Mukonka VM, Kamuliwo M, Macdonald MB, Haque U: Operational scale entomological intervention for malaria control: strategies, achievements and challenges in Zambia. Malaria J 2013, 12:10.

50. Gillies MT, Demeillon B: The Anophelinae of Africa South of the Sahara (Ethiopian zoogeographical region). Johannesburg: South African Institute of Medical Research; 1968.

51. Burkot TR, Williams JL, Schneider I: Identification of Plasmodium falciparum-infected mosquitoes by a double antibody enzyme-linked immunosorbent assay. AmJTrop Med Hyg 1984, 33:783-788.

52. Scott JA, Brogdon WG, Collins FH: Identification of single specimens of the Anopheles gambiae complex by the polymerase chain reaction. AmJTrop Med Hyg 1993, 49:520-529.

53. Koekemoer LL, Lochouarn L, Hunt RH, Coetzee M: Single-strand conformation polymorphism analysis for identification of four members of the Anopheles funestus (Diptera: Culicidae) group. J Med Entomol 1999, 36:125-130.

54. Durnez L, Van Bortel W, Denis L, Roelants $P$, Veracx A, Trung HD, Sochantha $T$, Coosemans M: False positive circumsporozoite protein ELISA: a challenge for the estimation of the entomological inoculation rate of malaria and for vector incrimination. Malaria J 2011, 10:195.

55. Dusfour I, Carinci R, Gaborit P, Issaly J, Girod R: Evaluation of four methods for collecting malaria vectors in French Guiana. J Econ Entomol 2010, 103:973-976.

56. Githeko AK, Service MW, Mbogo CM, Atieli FA, Juma FO: Sampling Anopheles arabiensis, A. gambiae sensu lato and A. funestus (Diptera: Culicidae) with CDC light-traps near a rice irrigation area and sugar can belt in western Kenya. Bull Entomol Res 1994, 84:319-321.

57. Sithiprasasna $R$, Jaichapor B, Chanaimongkol S, Khongtak $P$, Lealsirivattanakul T, Tiang-Trong S, Burkett DA, Perich MJ, Wirtz RA, Coleman RE: Evaluation of candidate traps as tools for conducting surveillance for Anopheles mosquitoes in a malaria-endemic area in western Thailand. $J$ Med Entomol 2004, 41:151-157.

58. Dia I, Diallo D, Duchemin JB, Ba Y, Konate L, Costantini C, Diallo M: Comparisons of human-landing catches and odor-baited entry traps for sampling malaria vectors in Senegal. J Med Entomol 2005, 42:104-109.

59. Stoops CA, Gionar YR, Rusmiarto S, Susapto D, Andris H, Elyazar IR, Barbara KA, Munif A: Laboratory and field testing of bednet traps for mosquito (Diptera: Culicidae) sampling in West Java, Indonesia. J Vector Ecol 2010, 35:187-196.

60. Duo-quan W, Lin-hua T, Zhen-cheng G, Xiang Z, Man-ni Y, Wei-kang J: Comparative evaluation of light-trap catches, electric motor mosquito catches and human biting catches of Anopheles in the Three Gorges Reservoir. PLoS One 2012, 7:e28988.

61. Sinka ME, Bangs MJ, Manguin S, Coetzee M, Mbogo CM, Hemingway J, Patil AP, Temperley WH, Gething PW, Kabaria CW, et al: The dominant Anopheles vectors of human malaria in Africa, Europe and the Middle East: occurrence data, distribution maps and bionomic precis. Parasit Vectors 2010, 3:117.

62. Sinka ME, Bangs MJ, Manguin S, Rubio-Palis Y, Chareonviriyaphap T, Coetzee M, Mbogo CM, Hemingway J, Patil AP, Temperley WH, et al: A global map of dominant malaria vectors. Parasit Vectors 2012, 5:69. 
63. Seyoum A, Sikaala CH, Chanda J, Chinula D, Ntamatungiro AJ, Hawela M, Miller JM, Russell TL, Briet OJ, Killeen GF: Human exposure to anopheline mosquitoes occurs primarily indoors, even for users of insecticidetreated nets in Luangwa Valley, South-east Zambia. Parasit Vectors 2012, 5:101.

64. Okumu FO, Moore J, Mbeyela E, Sherlock M, Sangusangu R, Ligamba G, Russell T, Moore SJ: A modified experimental hut design for studying responses of disease-transmitting mosquitoes to indoor interventions: the Ifakara experimental huts. PLoS One 2012, 7:e30967.

65. Okumu FO, Mbeyela E, Lingamba G, Moore J, Ntamatungiro AJ, Kavishe DR, Kenward MG, Turner E, Lorenz LM, Moore SJ: Comparative field evaluation of combinations of long-lasting insecticide treated nets and indoor residual spraying, relative to either method alone, for malaria prevention in an area where the main vector is Anopheles arabiensis. Parasit Vectors 2013, 6:46.

66. Maia MF, Robinson A, John A, Mgando J, Simfukwe E, Moore SJ: Comparison of the CDC Backpack aspirator and the Prokopack aspirator for sampling indoor- and outdoor-resting mosquitoes in southern Tanzania. Parasit Vectors 2011, 4:124.

67. Pates HV, Takken W, Curtis CF, Huisman PW, Akinpelu O, Gill GS: Unexpected anthropophagic behaviour in Anopheles quadriannulatus. Med Vet Entomol 2001, 15:293-298.

68. Torr SJ, Della Torre A, Calzetta M, Costantini C, Vale GA: Towards a fuller understanding of mosquito behaviour: use of electrocuting grids to compare the odour-orientated responses of Anopheles arabiensis and An. quadriannulatus in the field. Med Vet Entomol 2008, 22:93-108.

69. Huho B, Briet O, Seyoum A, Sikaala C, Bayoh N, Gimnig J, Okumu F, Diallo D, Abdulla S, Smith T, et al: Consistently high estimates for the proportion of human exposure to malaria vector populations occurring indoors in rural Africa. Int J Epidemiol 2013. doi:10.1093/ije/dys214.

70. Ngufor C, N'Guessan R, Boko P, Odjo A, Vigninou E, Asidi A, Akogbeto M, Rowland $\mathrm{M}$ : Combining indoor residual spraying with chlorfenapyr and long-lasting insecticidal bed nets for improved control of pyrethroidresistant Anopheles gambiae: an experimental hut trial in Benin. Malaria J 2011, 10:343.

71. Briet OJ, Smith TA, Chitnis N: Measurement of overall insecticidal effects in experimental hut trials. Parasit Vectors 2012, 5:256.

doi:10.1186/1756-3305-6-91

Cite this article as: Sikaala et al: Evaluation of alternative mosquito sampling methods for malaria vectors in Lowland South - East Zambia.

Parasites \& Vectors 2013 6:91.

\section{Submit your next manuscript to BioMed Central and take full advantage of:}

- Convenient online submission

- Thorough peer review

- No space constraints or color figure charges

- Immediate publication on acceptance

- Inclusion in PubMed, CAS, Scopus and Google Scholar

- Research which is freely available for redistribution 